\title{
Lymphatic vessel development: fluid flow and valve-forming cells
}

\author{
Tsutomu Kume \\ Feinberg Cardiovascular Research Institute, Department of Medicine, Northwestern University School of Medicine, Chicago, Illinois, USA.
}

\begin{abstract}
Hemodynamic forces regulate many aspects of blood vessel disease and development, including susceptibility to atherosclerosis and remodeling of primary blood vessels into a mature vascular network. Vessels of the lymphatic circulatory system are also subjected to fluid flow-associated forces, but the molecular and cellular mechanisms by which these forces regulate the formation and maintenance of lymphatic vessels remain largely uncharacterized. This issue of the $J C l$ includes two articles that begin to address how fluid flow influences lymphatic vessel development and function. Sweet et al. demonstrate that lymph flow is essential for the remodeling of primary lymphatic vessels, for ensuring the proper distribution of smooth muscle cells (SMCs), and for the development and maturation of lymphatic valves. Kazenwadel et al. show that flow-induced lymphatic valve development is initiated by the upregulation of GATA2, which has been linked to lymphedema in patients with Emberger syndrome. Together, these observations and future studies inspired by these results have potential to lead to the development of strategies for the treatment of lymphatic disorders.
\end{abstract}

\section{The lymphatic vascular system} in health and disease

The lymphatic vascular system is essential for lipid absorption/transport from the digestive system, maintenance of tissue fluid homeostasis, and immune surveillance (1). This system also participates in many pathological processes, such as inflammatory disease, tumor metastasis, and atherosclerosis (2). Deficiencies in lymphatic vessels can impede lymph drainage, which causes lymph to accumulate in the interstitial tissue and can lead to lymphedema. In mouse embryos, development of the lymphatic vascular system begins shortly after blood circulation is established (3) and is triggered when a subpopulation of venous cells become specified for differentiation into lymphatic endothelial cells (LECs) via the upregulation of PROX1, a master regulator of the lymphatic vas- cular phenotype. At approximately E9.5, $\mathrm{PROX}^{+}$lymphatic endothelial progenitors bud off and migrate from the anterior cardinal and intersomitic veins, and then differentiate into LECs, which form the lymph sacs and superficial lymphatic vessels (3). As LECs proliferate and sprout, the resulting lymphatic vessels develop into the primary lymphatic network. At E15.5-E16, this primary lymphatic plexus begins to remodel and mature, forming a hierarchical lymphatic vascular network composed of lymphatic capillaries, which are highly permeable, and collecting lymphatic vessels, which contain smooth muscle cells (SMCs) and intraluminal valves. The valves prevent lymph backflow, and recent studies demonstrate that one of the key events that initiate lymphatic valve formation involves the upregulation of PROX1 and FOXC2 expression in clusters of lym-

\section{Related Articles: pp. 2979 and 2995}

Conflict of interest: The author has declared that no conflict of interest exists.

Reference information: / Clin Invest. 2015;125(8):2924-2926. doi:10.1172/JCI83189.

phatic valve-forming cells $(4,5)$. PROX1 and FOXC2 are believed to act in concert with mechanical forces to establish the territory for valve formation; however, the nature and the molecular intermediaries of these interactions have been difficult to characterize. This issue of the JCI features two articles, authored by Sweet et al. and Kazenwadel et al., that are among the first to directly address these topics of inquiry $(6,7)$.

\section{Lymph flow as a key regulator of lymphatic vessel development}

A small subpopulation of $\mathrm{PROX}^{+} \mathrm{LEC}$ progenitors remains in the veins and contributes to the formation of lymphovenous valves, which are located at the junction of the jugular and subclavian veins and prevent the backflow of blood into lymphatic vessels (8). Blackflow prevention also requires the formation of platelet thrombi at the lymphovenous junction, and thrombi formation is induced by binding of the mucin-type transmembrane protein podoplanin on the surface of LECs to the platelet-specific receptor C-type lectin-like receptor 2 (CLEC2). In Clec2-deficeint mice, the flow of lymph is impeded due to impaired thrombosis and blood backfills the lymphatic network; however, Clec2 deletion does not impair primary lymphatic vessel growth $(9,10)$. Thus, by employing mice harboring this mutation, Sweet et al. were able to study how lymph flow regulates maturation of the lymphatic vasculature in animals with developmentally normal primary lymphatic vessels but dysfunctional lymphatic flow. The results of Sweet and colleagues provide a powerful in vivo demonstration that lymph flow is necessary for the development and maturation of lymphatic valves (ref. 7 and Figure 1). Furthermore, loss of flow impaired the upregulation of several essential transcription factors in mesenteric lymphatic valves, including FOXC2, PROX1, and 
A

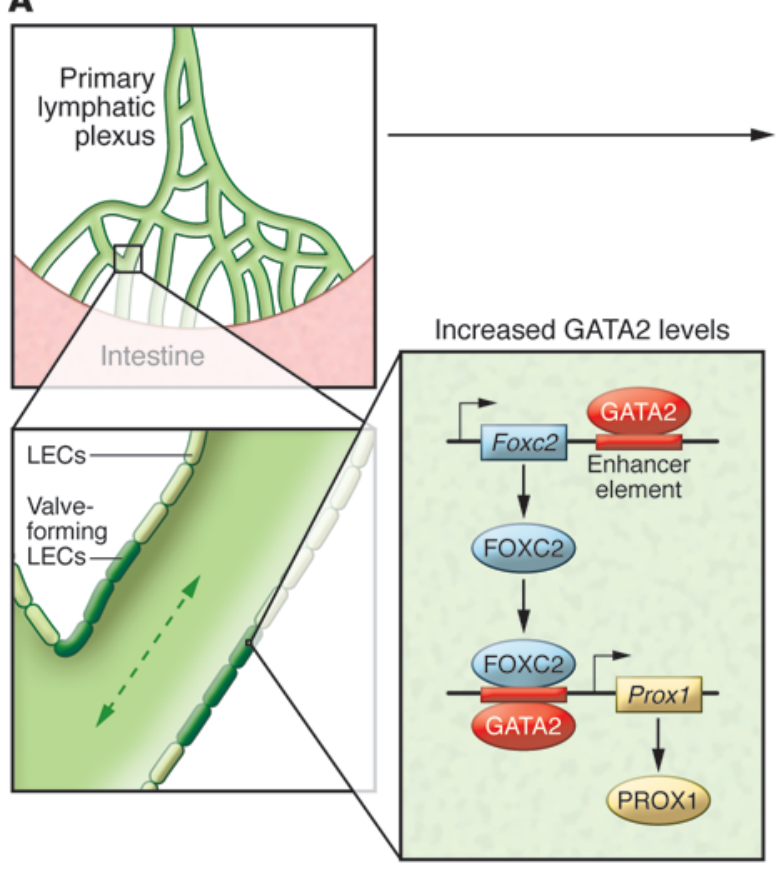

B

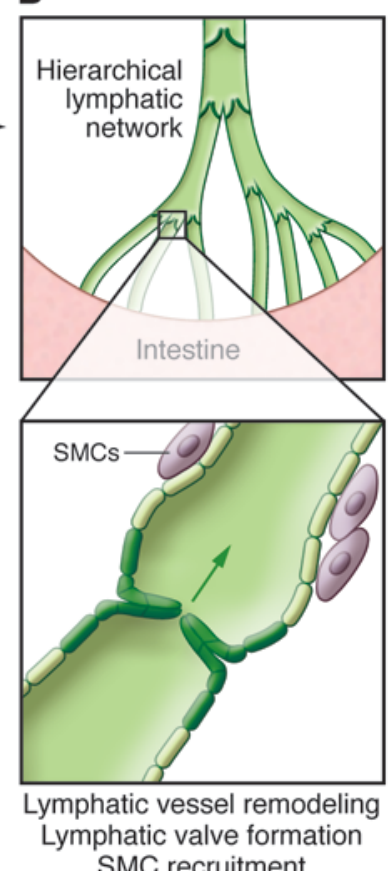

Figure 1. Mechanism of flow-dependent lymphatic vessel remodeling and valve formation in the developing mesentery. (A) Sites of vessel bifurcation in the primary lymphatic plexus produce turbulence in the flow of lymph (dashed arrow), which leads to an increase in the expression of GATA2. GATA2 is an upstream component of the mechanism by which the expression of FOXC2 and PROX1 in valve-forming cells establishes the site of valve formation. The studies by Kazenwadel et al. and Sweet et al. demonstrate that GATA2 is upregulated in response to shear stress and suggest that GATA2 binding at enhancer elements adjacent to PROX1- and FOXC2-encoding loci promotes expression of these known regulators of lymphatic development (inset). (B) Lymph flow (arrow) is also essential for lymphatic vessel remodeling and for proper recruitment of vascular SMCs (purple) to lymphatic collecting vessels.

allele may be the key characteristic that predisposes patients with Emberger syndrome to lymphedema. However, the enhancer analysis by Kazenwadel and colleagues was performed in vitro, so it would be useful to generate transgenic mice that express an enhancer-reporter construct to test whether activation of the GATA2-dependent enhancer element in the PROX1 locus occurs primarily in valve-forming cells and, consequently, whether the enhancer has a role in valve initiation. Kazenwadel et al. also identified an enhancer element positioned downstream of FOXC2 that is regulated by GATA2 activity. This observation, along with the results from similar analyses by Sweet et al. (7), corroborates GATA2 as the most upstream component of the mechanism by which shear stress regulates the expression of genes that control lymphatic valve development (Figure 1).

\section{Remaining questions and future directions}

The findings by Sweet et al. and Kazenwadel et al. raise a number of intriguing questions that will likely spur investigation into a variety of new topics. First, these studies provide convincing evidence that flow-induced lymphatic valve development is stimulated by GATA2 upregulation, and previous work has

and PROX1 are coexpressed in lymphovenous and lymphatic valves, as well scriptional regulator of valve develop ment (5). The affinity of this enhancer for GATA2 was reduced by the missense mutations that are associated with Emberger syndrome (R361L, C373R, and R396Q), suggesting that near or complete loss of function in one GATA2 
shown that the role of GATA2 in hematopoiesis and vascular integrity is controlled through a cis-regulatory element in the GATA2 locus (13); however, the regulatory elements that mediate the effect of shear stress on GATA2 expression have yet to be identified. Second, given that lymph flow is crucial for PROX1 upregulation during valve initiation in mice (7) - and that GATA2 and FOXC2, but not PROX1, are upregulated in cultured LECs by OSS $(5,7)$ - there appears to be an unknown mechanism that links shear stress, GATA2, and PROX1 in valve-forming cells in vivo. Third, GATA2, FOXC2, and NFATC1 comprise a complex regulatory network in the lymphatic system; for example, FOXC2 interacts with NFATC1, but not GATA2, and GATA2 does not bind NFATC1 $(5,6)$; this network, as well as the role of epigenetic modifications during the development of valve-forming cells, requires additional study. Finally, lymphatic and venous valve development are regulated by a number of common molecular pathways (16); thus, GATA2 may also have a role in venous valve development. Together, the findings from these investigations by Sweet et al. and Kazenwadel et al. expand our knowledge of both the blood and lymphatic vascular systems.

\section{Acknowledgments}

T. Kume is supported by grants from the $\mathrm{NIH} \mathrm{(EY019484} \mathrm{and} \mathrm{HL108795)} \mathrm{and}$ the Alberta Innovates - Health Solutions (AIHS) (Collaborative Research and Innovation Opportunities Team Grant).

Address correspondence to: Tsutomu Kume, Feinberg Cardiovascular Research Institute, Department of Medicine, Northwestern University School of Medicine, 303 E Chicago Ave., Chicago, Illinois 60611, USA. E-mail: t-kume@northwestern.edu.

1. Schulte-Merker S, Sabine A, Petrova TV. Lymphatic vascular morphogenesis in development, physiology, and disease. JCell Biol. 2011;193(4):607-618

2. Alitalo K. The lymphatic vasculature in disease. Nat Med. 2011;17(11):1371-1380.

3. Yang Y, Oliver G. Development of the mammalian lymphatic vasculature. JClin Invest. 2014;124(3):888-897.

4. Norrmen C, et al. FOXC2 controls formation and maturation of lymphatic collecting vessels through cooperation with NFATc1. JCell Biol. 2009;185(3):439-457.

5. Sabine A, et al. Mechanotransduction, PROX1, and FOXC2 cooperate to control connexin 37 and calcineurin during lymphatic-valve formation. Dev Cell. 2012;22(2):430-445.

6. Kazenwadel J, et al. GATA2 is required for lymphatic vessel valve development and maintenance. J Clin Invest. 2015;125(8):2979-2994.

7. Sweet DT, et al. Lymph flow regulates collecting lymphatic vessel maturation in vivo. JClin Invest. 2015;125(8):2995-3007.

8. Srinivasan RS, Oliver G. Prox1 dosage controls the number of lymphatic endothelial cell progenitors and the formation of the lymphovenous valves. Genes Dev. 2011;25(20):2187-2197.

9. Hess PR, et al. Platelets mediate lymphovenous hemostasis to maintain blood-lymphatic separation throughout life. JClin Invest. 2014;124(1):273-284.

10. Osada M, et al. Platelet activation receptor CLEC-2 regulates blood/lymphatic vessel separation by inhibiting proliferation, migration, and tube formation of lymphatic endothelial cells. J Biol Chem. 2012;287(26):22241-22252.

11. Petrova TV, et al. Defective valves and abnormal mural cell recruitment underlie lymphatic vascular failure in lymphedema distichiasis. Nat Med. 2004;10(9):974-981.

12. Spinner MA, et al. GATA2 deficiency: a protean disorder of hematopoiesis, lymphatics, and immunity. Blood. 2014;123(6):809-821.

13. Johnson KD, et al. Cis-element mutated in GATA2-dependent immunodeficiency governs hematopoiesis and vascular integrity. J Clin Invest. 2012;122(10):3692-3704.

14. Lim KC, et al. Conditional Gata2 inactivation results in HSC loss and lymphatic mispatterning. J Clin Invest. 2012;122(10):3705-3717.

15. Kazenwadel J, et al. Loss-of-function germline GATA2 mutations in patients with MDS/AML or MonoMAC syndrome and primary lymphedema reveal a key role for GATA2 in the lymphatic vasculature. Blood. 2012;119(5):1283-1291.

16. Bazigou E, Makinen T. Flow control in our vessels: vascular valves make sure there is no way back. Cell Mol Life Sci. 2013;70(6):1055-1066. 